\title{
Expression of macrophage migration inhibitory factor and its receptor CD74 in systemic sclerosis
}

\author{
CHRISTIAN JOHANA BAŃOS-HERNÁNDEZ ${ }^{1,2}$, RICHARD BUCALA ${ }^{3}$, JORGE HERNÁNDEZ-BELLO ${ }^{1,4}$, \\ JOSÉ EDUARDO NAVARRO-ZARZA', MARTHA ARISBETH VILLANUEVA-PÉREZ ${ }^{6}$, \\ MARISOL GODÍNEZ-RUBÍ, ISELA PARRA-ROJAS ${ }^{2}$, MIRNA VÁZQUEZ-VILLAMAR', \\ ANA LAURA PEREIRA-SUÁREZ', JOSÉ FRANCISCO MUŃOZ VALLE $E^{1,4}$
}

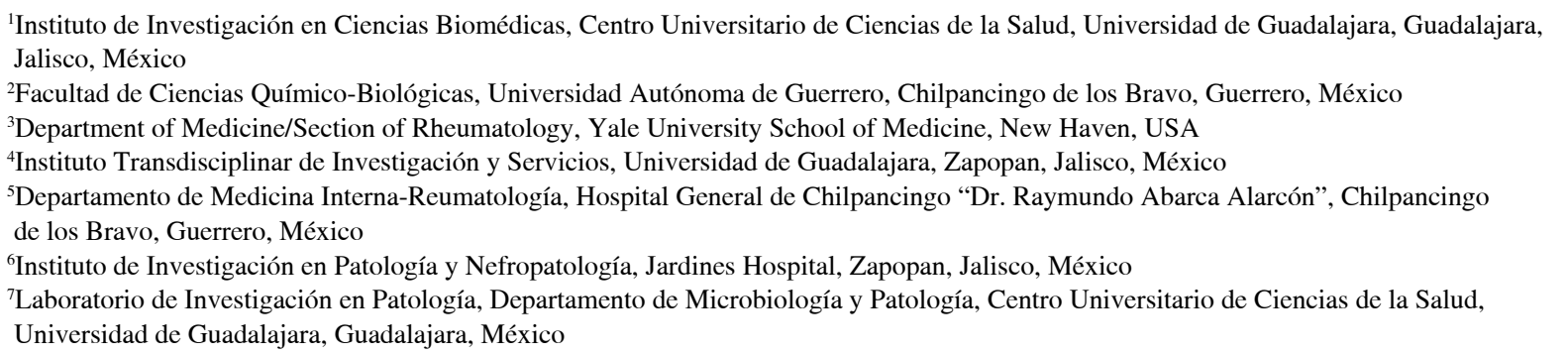

\begin{abstract}
Macrophage migration inhibitory factor (MIF) has been associated with the pathogenesis of several rheumatic diseases. In systemic sclerosis (SSc) it has been shown that MIF expression is dysregulated in serum and skin. However, the MIF receptor, CD74, has been poorly investigated and its potential role in the pathogenesis of SSc remains unknown. This study aimed to analyze mRNA, tissue, and serum expression of MIF and CD74 in patients with limited (lcSSc) and diffuse (dcSSc) systemic sclerosis. A case-control study in 20 SSc patients and 20 control subjects (CS) from southern México was conducted. MIF and CD74 mRNA expression levels were quantified by real-time PCR, MIF serum levels were measured by an ELISA kit, and MIF and its receptor CD74 were evaluated by immunohistochemistry of skin biopsies. MIF mRNA expression was significantly higher in CS than in SSc patients $(p=0.02)$, while CD74 showed no differences between patients and CS. MIF serum levels were similar between SSc patients and CS: $d c S S c=3.82 \mathrm{ng} / \mathrm{ml}, \mathrm{lcSSc}=3.57 \mathrm{ng} / \mathrm{ml}$, and $C S=3.28 \mathrm{ng} / \mathrm{ml}$. In skin biopsies of SSc, MIF and CD74 were enhanced in keratinocytes, while they showed decreased expression in endothelial cells. On the other hand, the staining of CD74 was high in fibroblasts of dcSSc patients. Our findings show MIF and CD74 deregulation at the transcriptional and translational levels in SSc, which might be associated with the proinflammatory process leading to tissue remodeling and excessive fibrosis in SSc.
\end{abstract}

Key words: immunohistochemistry, skin, MIF, sclerosis systemic, CD74.

(Cent Eur J Immunol 2021; 46 (3): 375-383)

\section{Introduction}

Systemic sclerosis ( $\mathrm{SSc}$ ) is a multisystem autoimmune disease characterized by immune system activation, vascular injury, and tissue fibrosis of the skin and specific internal organs [1]. This disease can be classified into two disease subsets based on the extent of skin involvement: diffuse cutaneous systemic sclerosis (dcSSc) and limited cutaneous systemic sclerosis (lcSSc) [2]. The prevalence of SSc in the USA has been calculated as approximately 250 persons per million inhabitants [3]. In contrast, in the Mexican population, the overall prevalence of scleroderma has been reported at $0.02 \%$ [4]. The triggering agents of SSc to date are unknown; however, it has been considered a multifactorial disease with genetic, infectious, and environmental risk factors [5].

Emerging evidence suggests that the macrophage migration inhibitory factor (MIF), a pleiotropic inflammatory cytokine with a broad range of immunomodulatory properties, could play a role in the pathogenesis of SSc, but the exact mechanism is unclear. In 2003, Selvi et al. reported high serum concentrations of MIF in dcSSc patients and the MIF expression in skin biopsies of SSc patients [6]. On the other

Correspondence: José Francisco Muńoz Valle, PhD, Instituto de Investigación en Ciencias Biomédicas, Centro Universitario de Ciencias de la Salud, Universidad de Guadalajara, Guadalajara, Jalisco, México, e-mail: biologiamolecular@hotmail.com Submitted: 24.08.2020; Accepted: 10.05.2021 
hand, Becker et al. reported that MIF is increased in patients with pulmonary hypertension and recurrent digital ulcers, which can contribute to inflammation and vasculopathy in the SSc [7]. Additionally, it was reported that MIF stimulates the process of excessive fibrosis in SSc, increasing the proliferation of fibroblasts and collagen synthesis [8], and decreasing the apoptosis of dermal fibroblasts [9].

The involvement of the MIF receptor, CD74, is poorly known in SSc. CD74 is a molecule associated with the processing of MHC class II proteins, and it is a high-affinity cell membrane receptor for MIF $\left(\sim 9 \times 10^{-9} \mathrm{Kd}\right)[10]$. A small proportion of CD74 is modified by the addition of chondroitin sulfate (CD74-CS), and this form of CD74 is expressed on the cell surface [11]. However, MIF signaling through CD74 depends on the interaction with co-receptors, e.g. CD44, which, through their cytoplasmic domains, can recruit the necessary components to activate signaling $[12,13]$. Corallo et al. evaluated MIF and its receptors CD74/CD44 by immunohistochemistry (IHC) on skin biopsies from patients with SSc. They confirmed that MIF is abundant in both the serum and the skin of SSc patients, unlike the MIF receptors CD74/CD44, which showed no differences between patients and controls [14]. However, there are no reports that confirm this finding.

Recently, we observed that polymorphisms and functional haplotypes of the $M I F$ gene are associated with susceptibility to SSc and high MIF mRNA expression in a Mexican-Mestizo population from southern Mexico. In the same way, we found that MIF is associated with a proinflammatory response in SSc, as it correlates positively with the Th1 [interferon $\gamma($ IFN- $\gamma$ ) and tumor necrosis factor $\alpha$ (TNF- $\alpha$ )] and Th17 [interleukin (IL)-17A, IL-17F, IL-1 $\beta$, and IL-21] cytokine profiles [15]. Based on our previous results and the existing data, this study aimed to analyze whether there was an association between the expression of MIF and its binding receptor (CD74) at the systemic (mRNA and protein) and in situ (skin cells) levels in SSc patients.

\section{Material and methods Patients}

This study was conducted in $20 \mathrm{SSc}$ patients classified according to the 2013 American College of Rheumatology/European League Against Rheumatism classification criteria for SSc [16]. They were consecutively enrolled from the Rheumatology Department at Hospital General de Chilpancingo "Dr. Raymundo Abarca Alarcón", Chilpancingo de los Bravo, State of Guerrero, Mexico. In the same way, 20 control subjects (CS) recruited from the general population were included.

Informed written consent was obtained from all subjects before enrollment in the study. The investigation was performed according to the ethical guidelines of the 2013 Declaration of Helsinki. It was approved by the ethical, research, and biosecurity committee of the Hospital General de Chilpancingo “Dr. Raymundo Abarca Alarcón”, Chilpancingo de los Bravo, State of Guerrero, Mexico (CI/317/2016).

\section{MIF and CD74 mRNA expression analysis}

The peripheral blood sample was collected in EDTA blood collection tubes (BD Vacutainer NJ, USA). The total leukocytes were isolated using dextran reagent (5\%) (Sigma Aldrich Co), and the total RNA was obtained using TRIZOL reagent (Invitrogen, Carlsbad, CA, USA) conforming to the Chomczynski and Sacchi method [17]. The RNA concentration and purity were measured by spectrophotometry (ratio A260/A280) (NanoDrop 2000, Thermo Scientific). After that, the cDNA was synthesized from $1 \mu \mathrm{g}$ of total RNA, and reverse transcription was performed using primer oligodT (Promega Corporation, USA) as indicated by the manufacturer.

We conducted the quantification of $M I F$ and $G A P D H$ mRNA by real-time PCR, using UPL hydrolysis probes (Cat. No. 05190541001 and 05190541001, Roche Applied Science, Penzberg, Germany). All samples were run in triplicate using the conditions indicated in the UPL Gene Expression Assay protocol in a LightCycler 96 System (Roche Applied Science).

CD74 mRNA expression was quantified using TaqMan probes (Cat. No. 4331182, Applied Biosystems, United States), and GAPDH (Cat. No. 4331182, Applied Biosystems, United States) was used as a reference gene. The mRNA expression analysis was performed through $2^{-\Delta \Delta \mathrm{Cq}}$ and $2^{-\Delta \mathrm{Cq}}$ methods after validation of reaction efficiency for the target genes (MIF and $C D 74)$ and the reference gene $(G A P D H)$ [18].

\section{MIF serum levels}

The blood samples of all individuals were collected and centrifuged to obtain the sera, which were subsequently stored at $-20^{\circ} \mathrm{C}$. After that, MIF serum levels were determined by the Human MIF ELISA Kit Protocol (LEGEND MAX Human Active MIF ELISA Kit, BioLegend) according to the manufacturer's instructions. MIF assay sensitivity was $6 \mathrm{pg} / \mathrm{ml}$.

\section{Immunohistochemistry for MIF and CD74}

We analyzed the expression of MIF and CD74 in skin biopsy samples. Skin biopsies were obtained at the time of sera collection and were fixed in Michel's solution for later paraffin embedding. The skin samples were obtained by a $4-\mathrm{mm}$ puncture biopsy under local anesthesia from the affected skin. Samples were embedded in paraffin and cut into $5 \mu \mathrm{m}$ sections for mounting on pre-loaded slides.

Tissues were routinely processed by heat, xylene, and graded ethanol solutions. Once tissue sections were rehydrated, antigen retrieval was accomplished in a bath of sodium citrate solution $10 \mathrm{mM}(\mathrm{pH}=6)$ at $95^{\circ} \mathrm{C}$ for $10 \mathrm{~min}$ 
followed by cooling in a cold citrate solution. Endogenous peroxidase activity was neutralized with $\mathrm{H}_{2} \mathrm{O}_{2}$ (Peroxidase Block) for 10 minutes. To reduce the non-specific binding of primary antibody and polymer, the Novocastra Protein Block was applied. The sections of each biopsy were incubated overnight at $4{ }^{\circ} \mathrm{C}$ with one of the following primary antibodies: anti-MIF (Cat. ab55445; dilution $1: 200$ ) or anti-CD74 LN2 (Cat. ab9514; dilution $1: 200$ ). The detection of primary antibodies was performed using the polymer Novolink (Cat: RE7140-CE, Novocastra, UK). Sections were further incubated with the substrate/ chromogen, 3,3'-diaminobenzidine (DAB), prepared from DAB Chromogen and Novolink DAB Substrate Buffer. Finally, sections were counterstained with hematoxylin.

After staining, images of the slides were captured with a digital camera (Axiocam ICc 1; Zeiss AG, Oberkochen, Germany) and the reaction was assessed qualitatively by intensity of the mark. The analysis was performed by two independent pathologists in a blinded fashion to ensure consistent and reproducible results. The positive cells for the brand (CD74 or MIF) were quantified according to their lineage (keratinocytes, lymphocytes, fibroblasts, endothelium and salivary glands), based on their morphology and tissue location. In accordance with the Human Protein Atlas [19], we used human tissues in which CD74 or MIF are constitutively expressed (appendix and tonsil normal tissue for CD74; breast and prostate normal tissue for MIF, data not shown). The negative control for both antibodies was obtained by incubating the tissues in the absence of primary antibody.

\section{Statistical analysis}

Data were analyzed as follows: the qualitative variables were expressed as percentages and absolute frequency, and continuous variables distributed normally were expressed as the mean \pm standard deviation (SD). Those non-normally distributed were expressed as median and $5-95^{\text {th }}$ centiles. The Mann-Whitney $U$-test was used to evaluate differences between the two groups, and the Kruskal-Wallis test was used to analyze differences between three or more groups (for variables distributed non-normally) followed by Dunn's adjustment for multiple comparisons. Statistical analysis was performed using STATA version 11.1 and GraphPad Prism version 6.0 Software. A probability $(p)$ value of less than 0.05 was considered significant.

\section{Results}

\section{Clinical and demographic characteristics of SSc patients and CS}

The demographic and clinical data of SSc patients and CS are shown in Table 1. In this study, a total of 20 patients diagnosed with SSc (16 females and 4 males; aged $44.4 \pm 17.7$ years), and $20 \mathrm{CS}$ ( 15 females and 5 males;
Table 1. Demographic and clinical characteristics of systemic sclerosis $(\mathrm{SSc})$ patients

\begin{tabular}{lcc}
\hline Variables & SSc $(\boldsymbol{n}=\mathbf{2 0})$ & CS $(\boldsymbol{n}=\mathbf{2 0})$ \\
\hline Demographics & & \\
\hline Age $(\text { years })^{\mathrm{a}}$ & $44.4 \pm 17.7$ & $39.6 \pm 14.6$ \\
\hline \begin{tabular}{l} 
Gender \\
\hline Female
\end{tabular} & $80(16)$ & $75(15)$ \\
\hline Male & $20(4)$ & $25(5)$ \\
\hline BMI $\left(\mathrm{kg} / \mathrm{m}^{2}\right)^{\mathrm{a}}$ & $24.4 \pm 3.9$ & $26.9 \pm 4.0$ \\
\hline Clinical assessment & & \\
\hline \begin{tabular}{l} 
Disease subtype \\
\hline 1cSS
\end{tabular} & $70(14)$ & - \\
\hline dcSS $^{\mathrm{c}}$ & $30(6)$ & - \\
\hline Disease evolution (years) & $4(0.5-20)$ & - \\
\hline
\end{tabular}

\begin{tabular}{|c|c|c|}
\hline \multicolumn{3}{|l|}{ Clinical manifestations ${ }^{\mathrm{b}}$} \\
\hline Skin thickening & $95(19)$ & - \\
\hline Joint contractures & $95(19)$ & - \\
\hline Puffy fingers & $75(15)$ & - \\
\hline Raynaud's phenomenon & $70(14)$ & - \\
\hline Telangiectasia & $50(10)$ & - \\
\hline
\end{tabular}

\begin{tabular}{lcl}
\hline Clinical evaluation & & \\
\hline Spanish HAQ-DI, 0-3 scale $^{\mathrm{a}}$ & $0.46 \pm 0.44$ & - \\
\hline mRSS51 $^{\mathrm{c}}$ & $6(0-30)$ & - \\
\hline
\end{tabular}

\begin{tabular}{lll}
\hline Drug treatment $^{\mathrm{b}}$ & & \\
\hline None & $55(11)$ & $100(20)$ \\
\hline
\end{tabular}

\begin{tabular}{lll}
\hline Prednisone & $35(7)$ & - \\
\hline Methotrexate & $35(7)$ & - \\
\hline Chloroquine & $15(3)$ & -
\end{tabular}

$\overline{{ }^{a} \text { Data presented as mean } \pm \text { standard deviation. }{ }^{b} \text { Data presented as percentage }}$ and $n .{ }^{c}$ Data presented as median (p5-p95). SSc-systemic sclerosis, BMI-body mass index, dcSSc-diffuse cutaneous systemic sclerosis, lcSSc-limited cutaneous systemic sclerosis, mRSS - modified Rodnan skin score, Spanish HAQ-DI the Spanish version of Health Assessment Questionnaire Disability Index.

aged $39.6 \pm 14.6)$ were included. A higher percentage of individuals with a classification of $1 \mathrm{cSSc}(86 \%)$ compared to dcSSc (14\%) was observed in SSc patients.

SSc patients had disease evolution of 4 years (0.5-20) at the inclusion time. Most patients had sclerodactyly and joint contractures (95\%), followed by puffy fingers (75\%), Raynaud's phenomenon (70\%), and telangiectasia (50\%). Concerning the clinical evaluation, the patients had a mean HAQ (Health Assessment Questionnaire disability index) of $0.46 \pm 0.44$ and a median mRSS51 (Modified Rodnan Skin Score) of 6 (0-30). Most patients were not under treatment $(55 \%)$ because they were enrolled in the present study at the time of diagnosis by the rheumatologist. 
A

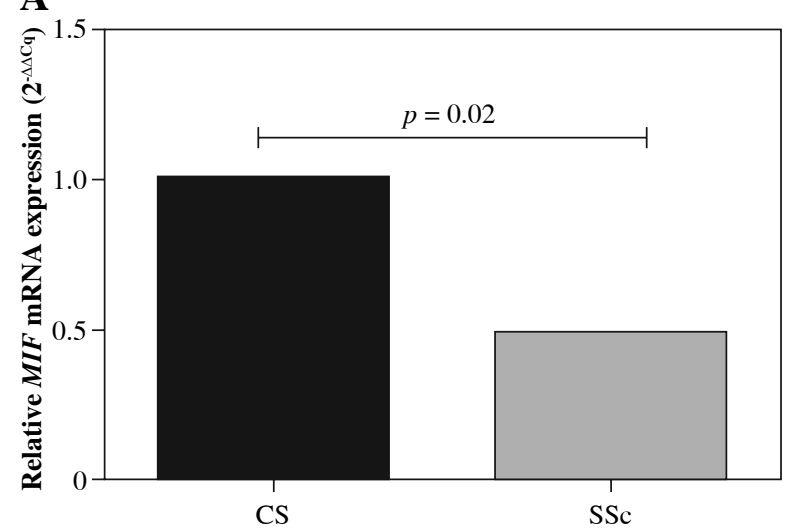

C

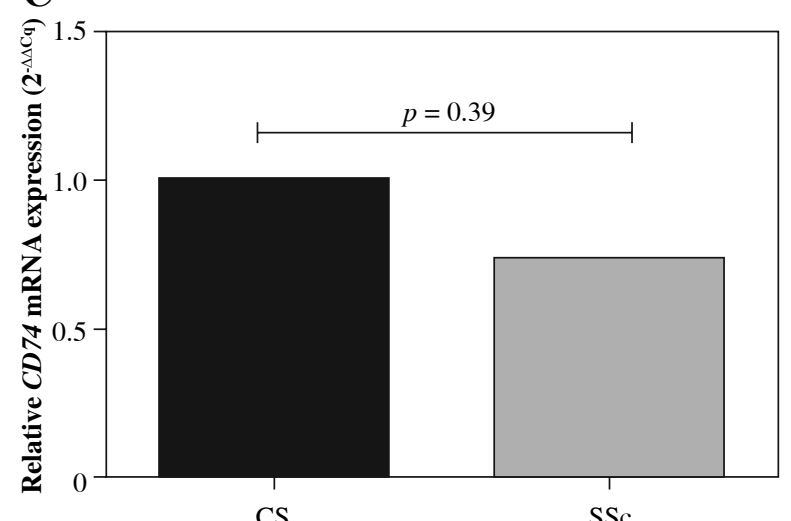

B

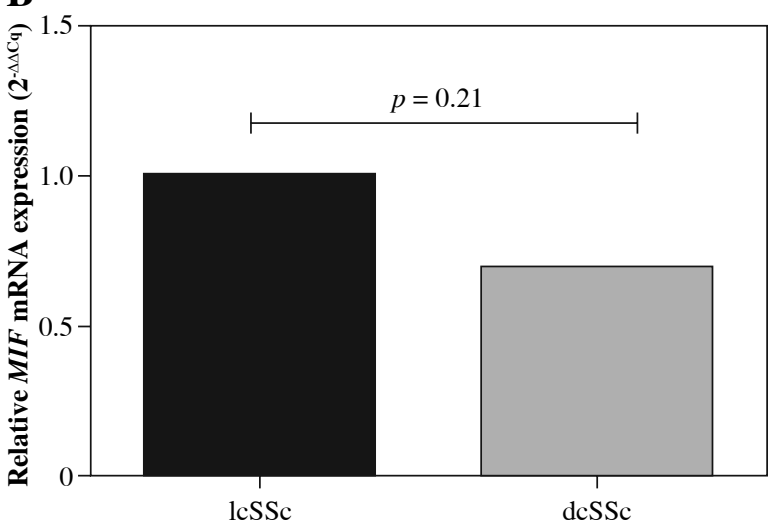

D

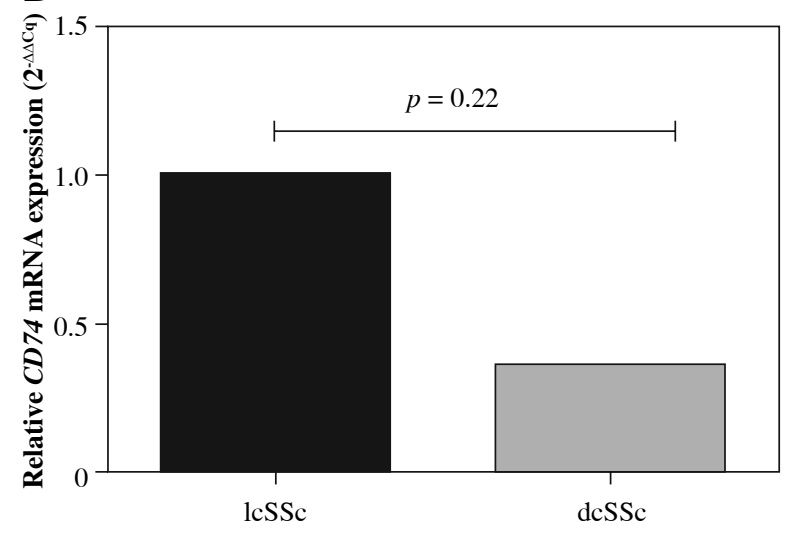

Fig. 1. Relative MIF and CD74 mRNA expression. MIF and CD74 mRNA expression in peripheral blood mononuclear cells from control subjects (CS) and systemic sclerosis (SSc) (A, C). MIF and CD74 mRNA expression in SSc patients with limited cutaneous (lcSSc) and diffuse cutaneous SSc $(\mathrm{dcSSc})(\mathbf{B}, \mathbf{D})$. Relative expression analysis was performed using $2^{-\Delta \mathrm{Cq}}$ and $2^{-\Delta \Delta \mathrm{Cq}}$ methods, and the GAPDH gene was used as the reference gene. Statistical comparisons between groups were made using the Mann-Whitney $U$ test

On the other hand, patients under treatment were treated mainly with methotrexate $(35 \%)$ and prednisone $(35 \%)$, followed by chloroquine (15\%).

\section{MIF and CD74 mRNA expression}

$M I F$ and $C D 74$ mRNA expression levels were evaluated in peripheral blood mononuclear cells from SSc patients and CS (Fig. 1A, C). According to the $2^{-\triangle \Delta C q}$ method, $M I F$ mRNA expression was 2-fold less in SSc compared to CS (Fig. 1A). Similarly, CD74 mRNA expression was lower (1.4-fold less) in SSc patients compared to CS (Fig. 1C). These differences were only significant for the MIF mRNA expression when the data were assessed by the $2^{-\Delta \mathrm{Cq}}$ method $(p=0.02)$ [20]. To investigate the role of MIF and CD74 according to the types of SSc, we compared the MIF (Fig. 1B) and $C D 74$ (Fig. 1C) mRNA expression between patients with lcSSc and dcSSc. We observed that patients with dcSSc had lower MIF (1.4-fold) and CD74 (2.6-fold) mRNA expression than patients with lcSSc, however, this difference was not statistically significant $(p>0.05)$.

\section{Serum levels of MIF according to the type of SSc}

We observed higher MIF serum levels in SSc patients in comparison to the CS group [SSc: 3.72 (0.58-9.5) ng/ml vs. CS: $3.28(0.74-4.37) \mathrm{ng} / \mathrm{ml}]$, but the difference was not statistically significant ( $p=0.52$, Fig. 2A). Subsequently, we compared the MIF serum levels between lcSSc and dcSSc patients, and CS (Fig. 2B) and observed higher MIF serum levels in dcSSc patients $(3.82 \mathrm{ng} / \mathrm{ml})$, followed by $1 \mathrm{lSSc}$ patients $(3.57 \mathrm{ng} / \mathrm{ml})$ and CS $(3.28 \mathrm{ng} / \mathrm{ml})$. However, these differences were not statistically significant $(p=0.77)$.

\section{Detection of MIF and CD74 in skin cells}

We analyzed the expression of MIF and CD74 in skin biopsies by IHC. Twenty cases of scleroderma skin were examined and immunostained with anti-MIF and anti-CD74 antibodies. All tissues showed a lack of epidermal appendages and scant cellularity of the reticular dermis, which showed thickened collagen bundles. In the epidermis, we found that most patients showed expression 
A

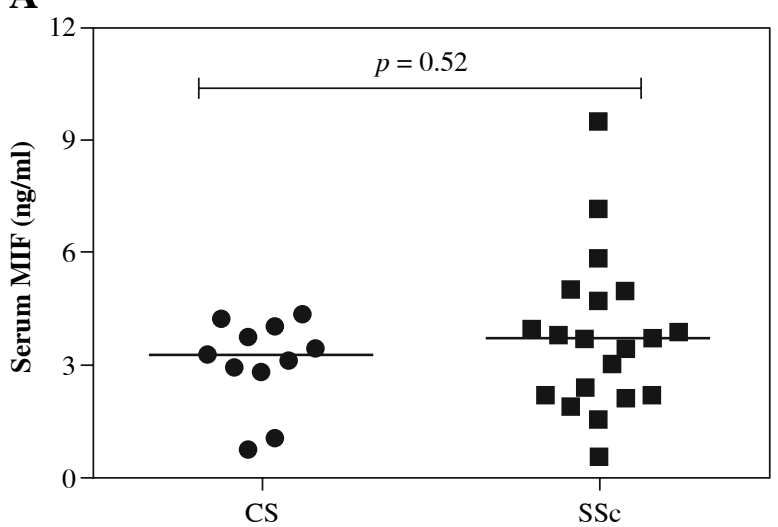

B

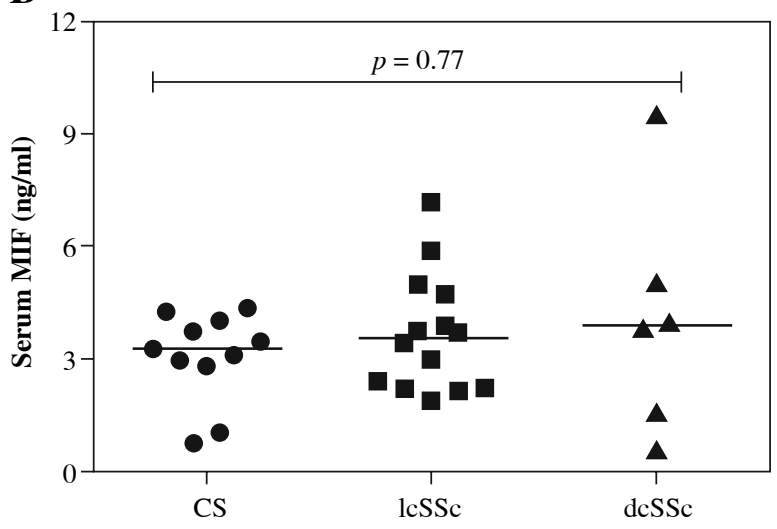

Fig. 2. MIF serum levels in control subjects (CS) and systemic sclerosis (SSc) patients. A) Comparison of soluble levels of MIF between CS and SSc patients. B) Comparison of soluble levels of MIF between CS, limited cutaneous (lcSSc), and diffuse cutaneous SSc (dcSSc). The values are shown as scatter diagrams. The $p$-value was calculated with the Kruskal-Wallis test or Mann-Whitney $U$ test, as appropriate

positive of MIF and CD74 in keratinocytes followed by the spindle fibroblast-like cells (Fig. 3C, D). Likewise, MIF and CD74 were expressed in dermal cells, such as glands and infiltrating mononuclear cells. On the other hand, in endothelial cells, both MIF and CD74 were expressed in a lower percentage (Fig. 3A, B). In the control skin specimen, MIF was strongly expressed in all layers of the epidermis and in endothelium and scarce fibroblasts of the papillary dermis. CD74 was irregularly expressed in some keratinocytes of the basal and spinosum stratum, and in endothelial cells (data not shown).

In the stratification of SSc subtypes (lcSSc and dcSSc), CD74 was more strongly stained in fibroblasts of dcSSc patients (Fig. 4B, C) than in lcSSc (Fig. 4B, D) $(p=0.001)$. Similarly, MIF showed stronger staining in fibroblasts of dcSSc (Fig. 4A) patients than in lcSSc patients, but these differences were not significant $(p=0.35)$.

\section{Discussion}

Macrophage migration inhibitory factor is an immunoregulatory cytokine that inhibits apoptosis induced by p53 activation and regulates the proliferation of different types of cells [21] via its cognate CD74 receptor and CD44 signaling co-receptor [22]. In SSc, it has been suggested that MIF could contribute to skin fibrosis by inhibiting the apoptosis of dermal fibroblasts [9], and it augments fibroblast proliferation and collagen synthesis [8]. However, the participation of MIF receptors in the pathogenesis of SSc has been poorly investigated [14]. To obtain more information about the role of MIF and CD74 in SSc, we evaluated the MIF serum levels in lcSSc, dcSSc, and CS, followed by the MIF and CD74 mRNA expression in leukocytes and the skin.

Corallo et al. suggest that MIF is produced by fibroblasts at the beginning of their differentiation into myofi- broblasts and that MIF probably prevents their apoptosis in SSc [14], and increased MIF expression has been reported by high-genotypic MIF expressing skin fibroblasts in culture [20]. It has also been suggested that MIF produced by activated $\mathrm{T}$ cells may exert an autocrine effect to inhibit apoptosis in fibroblasts, thereby promoting their survival, persistence, and proliferation [9]. The present study evaluated $M I F$ mRNA expression in peripheral blood leukocytes, where we observed higher MIF mRNA expression in CS than in SSc patients. Previous studies have reported that anti-rheumatic drugs such as chloroquine negatively regulate the mRNA expression of some inflammatory cytokines (TNF- $\alpha$ and IL-1 $\beta$ ), which correlate with MIF expression [23]. However, despite a significant percentage of our patients being under treatment, MIF mRNA expression was not different between SSc patients without or with drug treatment. To better understand the reasons for this discordance, the processes involved in the synthesis and degradation of MIF at the tissue and systemic levels should be investigated in future studies in SSc patients.

CD74 is a high-affinity receptor for MIF [24], and we evaluated the $C D 74$ mRNA expression in peripheral blood leukocytes. In this regard, higher mRNA expression of $C D 74$ in CS was observed in comparison with the SSc patients, similar to that found in MIF mRNA expression.

Several studies have shown increased MIF levels in serum and dermal fibroblast culture supernatant of SSc patients [7, 9, 25]. In serum, it has been observed that patients with dcSSc have higher levels of MIF than patients with $1 \mathrm{cSSc}$ and CS [6, 7]. However, in our population, these significant differences were not observed when comparing the serum levels of MIF between patients with SSc and CS, or when stratifying them by type of SSc. Our results coincide with those reported by Wu et al., who did not observe significant differences of MIF serum levels between types of 
A

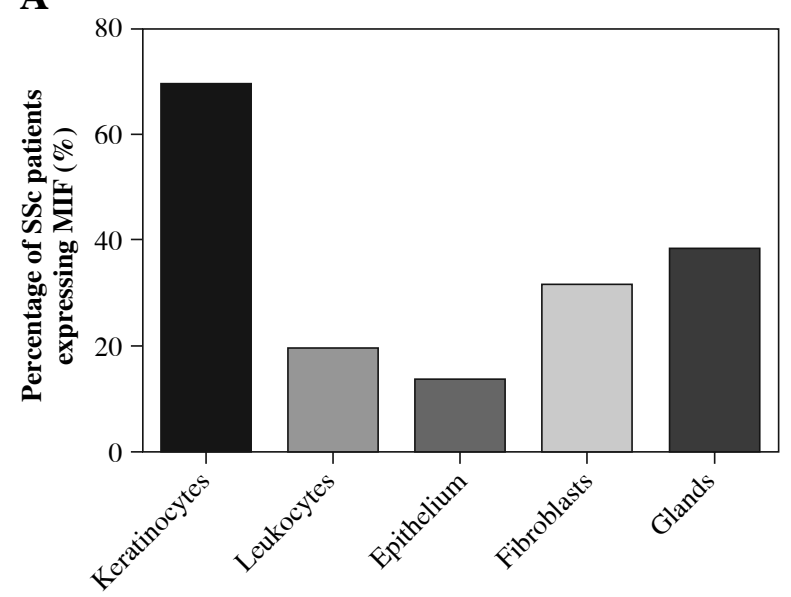

C

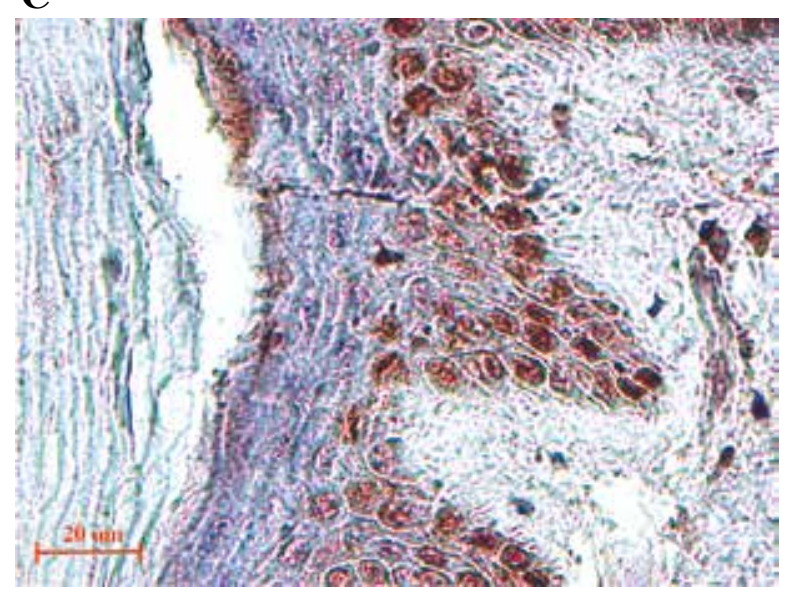

B

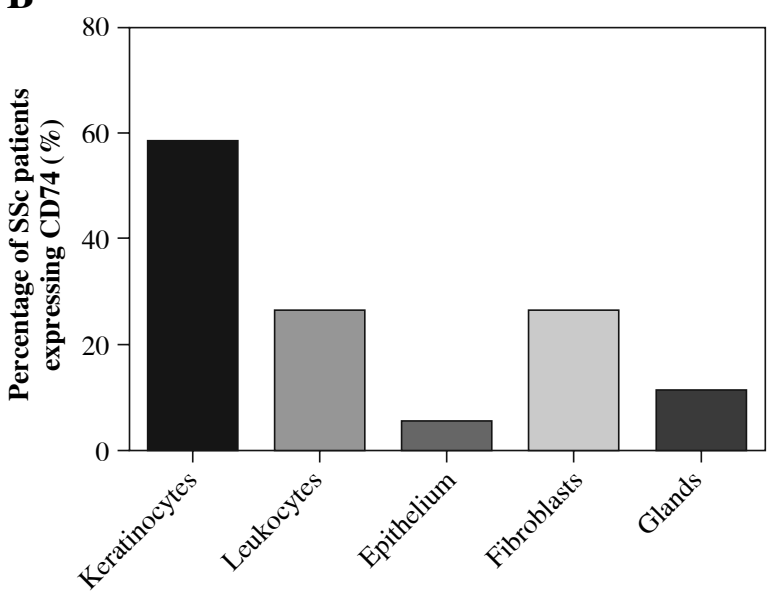

D

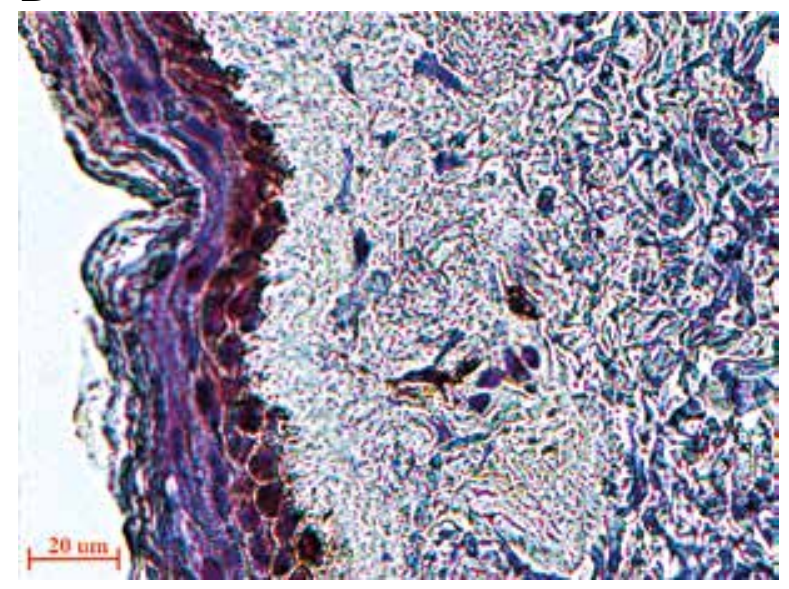

Fig. 3. MIF and CD74 expression in skin biopsies. A) Percentage of systemic sclerosis (SSc) patients with MIF staining in skin cells. B) Percentage of SSc patients with CD74 staining in skin cells. Images were qualitatively analyzed as positive and negative for each cell type by two specialist pathologists. Expression of MIF (C) and CD74 (D) in the skin of patients with SSc. Representative sections stained by immunohistochemistry (3,3'-diaminobenzidine, brown). In the stratum basale, MIF was expressed in the keratinocytes' cytoplasm and nucleus, and partially in the stratum spinosum. CD74 was expressed predominately in keratinocytes of the stratum basale

SSc; however, they found significantly higher MIF levels in the group of patients with SSc in comparison to the CS group [20]. The discrepancy between the previous studies reflects the variability in the serum levels of MIF in SSc, which can be influenced by the treatment (methotrexate, glucocorticoids, and chloroquine) [26, 27]. On the other hand, probably the serum levels of this protein do not correlate positively with their production at sites of skin pathology [28].

The expression of MIF mRNA in leukocytes showed no correlation with MIF serum levels in the present study. In this regard, it is known that the expression of the mRNA of a particular gene does not always predict the expression of the protein; this is especially true in the case of cytokines, and the correlation between the two can vary significantly [29]. There are several possible explanations for these differences between mRNA and protein levels, such as post-transcriptional regulation, which has been shown to play a vital role in controlling the expression of cytokines by modulating mRNA stability [30, 31]. However, it is important to consider that MIF is not only expressed in the blood cells but is also expressed in skin cells, and it is associated with wound repair [32]. MIF is also present in preformed intracellular stores and undergoes a specialized export process upon inflammatory stimulation [33].

A crucial role of MIF in wound healing has been documented in previous studies, where it mediates the response to tissue injury and regulates the immunological and inflammatory phases of the wound process [32, 34]. MIF also exerts chemotactic action on the keratinocytes of the skin; in addition, fibroblasts of the skin wound produce a more significant amount of MIF in response to inflam- 
A

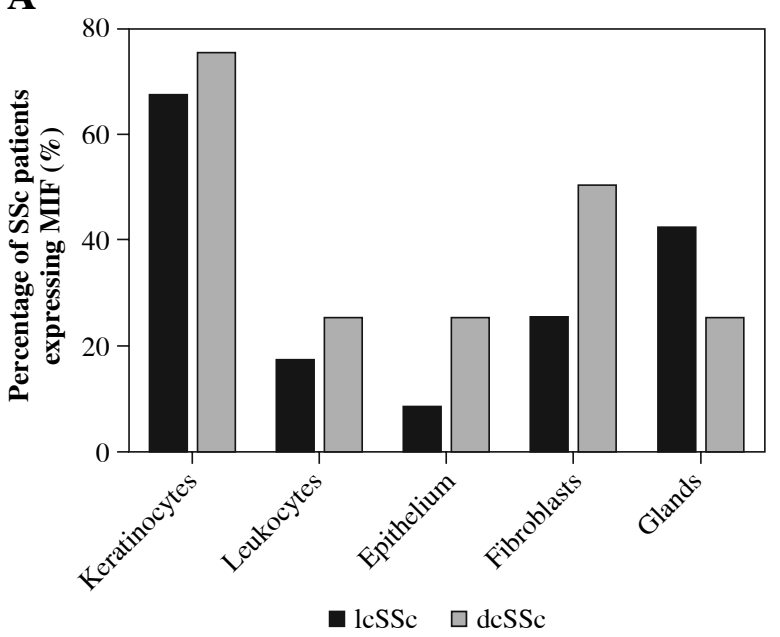

C

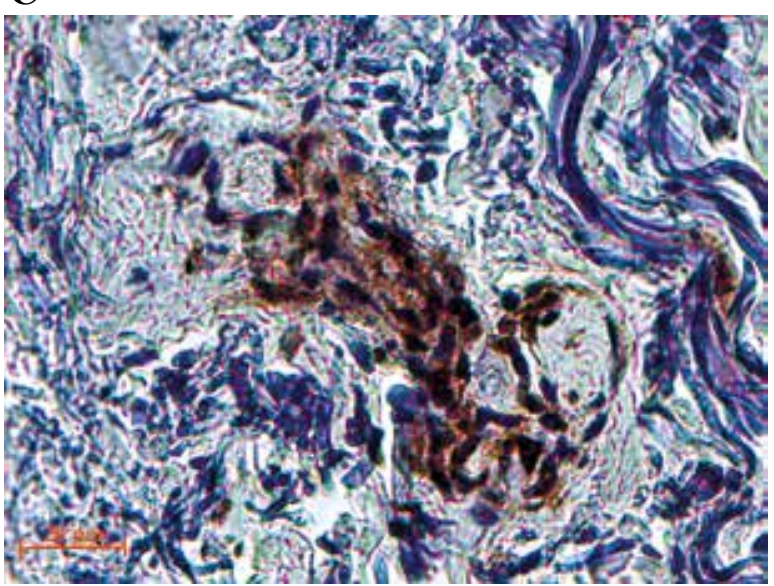

B

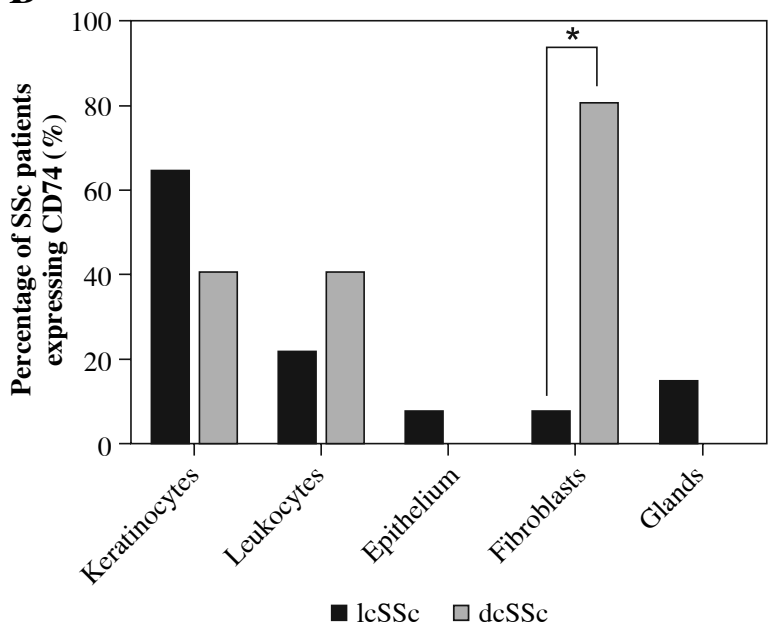

D

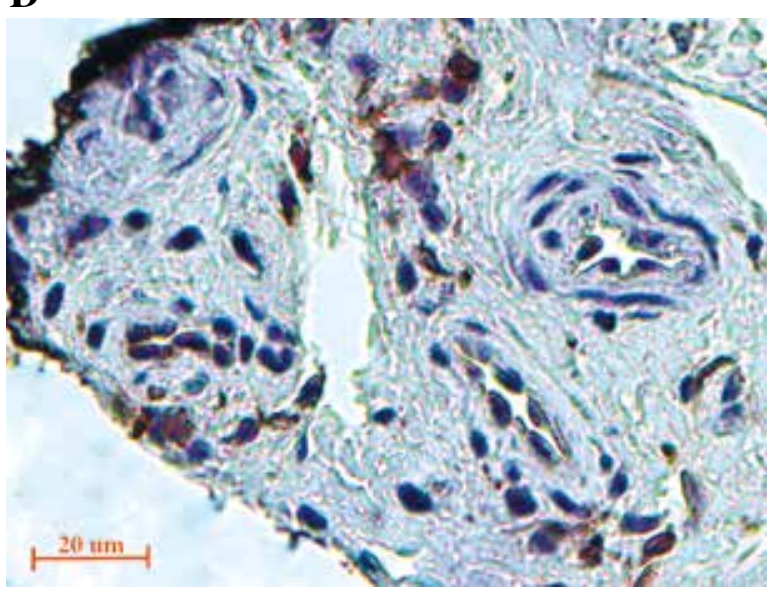

Fig. 4. MIF and CD74 expression in skin biopsies by type of systemic sclerosis (SSc). A) Comparison of the percentage of limited cutaneous (lcSSc) or diffuse cutaneous SSc (dcSSc) patients with MIF positive staining. B) Comparison of the percentage of lcSSc or dcSSc patients with CD74 positive staining. Expression of CD74 in fibroblasts of patients with dcSSc (C) and lcSSc (D). Representative sections stained by immunohistochemistry (3,3'-diaminobenzidine, brown. C) A group of fibroblasts adjacent to a capillary vessel in the reticular dermis shows strong expression of CD74 in the cytoplasm and nucleus of a dcSSc patient. D) There is moderate expression of CD74 in scattered fibroblasts and endothelial cells of the reticular dermis in the tissue of lcSSc patients. *Significant differences $(p<0.05)$ were observed between lcSSc and dcSSc when comparing the positivity of CD74 staining in fibroblasts. Images were qualitatively analyzed as positive and negative for each cell type by two specialist pathologists

matory stimulation, and interestingly, this factor is important in the migration of the fibroblasts and the regeneration of the skin after the wound $[35,36]$. Taken together, these results could suggest that MIF contributes to the wound healing process of skin tissue, including tissue remodeling and fibrosis, which are characteristic of SSc.

To obtain more information about the role of MIF and CD74 in SSc, we examined skin biopsies by IHC. We found that MIF and CD74 were expressed in a higher percentage in keratinocytes followed by fibroblasts, sweat glands, infiltrating mononuclear cells, and skin endothelial cells. These findings agree with those reported by Selvi et al., who observed MIF staining in keratinocytes, fibroblasts, sweat glands, infiltrating mononuclear cells, and endothelial cells; the presence of the CD74 receptor was not evaluated in their study [6].

Corallo et al. evaluated the presence of MIF and its CD74 receptor in skin biopsies of patients with SSc and also reported increased immunoreactivity of MIF in keratinocytes, fibroblasts, endothelium, sebaceous/sweat glands of lcSSc/dcSSc patients with skin involvement. They detected only weak immunoreactivity of MIF in the control subjects' skin and in the unaffected skin of patients with lcSSc. Whereas the CD74/CD44 receptor complex showed 
no difference between the control and affected and unaffected skin of dcSSc/lcSSc patients [14], MIF and CD74 expression in skin cells - mainly in keratinocytes and fibroblasts - supports the hypothesis that MIF and most likely CD74 play an essential role in the development of fibrotic lesions of the skin in the course of SSc. It is known that keratinocytes have an important role in the pathogenesis of SSc, through the activation of fibroblasts independently of transforming growth factor $\beta$ (TGF- $\beta$ ) [37]. Also, it has been reported that increased MIF expression may suppress apoptosis in keratinocytes [38].

Finally, we observed a higher percentage of CD74 expression in fibroblasts of dcSSc patients than in lcSSc patients. On the other hand, Corallo et al. did not find significant differences in the analysis of the CD74/CD44 receptors between the control and dcSSc/lcSSc affected and unaffected skin [14]. There also appeared to be a higher percentage of MIF expression in fibroblasts of dcSSc patients than in lcSSc patients; however, the differences between the types of SSc were not significant.

In conclusion, the present findings support the notion that MIF and CD74 have a more important role in the localized inflammatory process (in the skin) in SSc patients than at the systemic level since we observed lower MIF expression and CD74 mRNA in SSc patients than in CS. The expression of MIF and CD74 was found in the skin of SSc patients, mainly in keratinocytes and fibroblasts, which are essential cells in the tissue remodeling process in SSc. In addition, the overexpression of CD74 observed in biopsies of patients with dcSSc suggests that CD74 could be involved in exacerbated fibrosis in dcSSc; however, their participation is unclear. Additional studies are required in the different SSc types to describe the involvement of $\mathrm{MIF} / \mathrm{CD} 74$ in the fibrotic process of this disease.

\section{Acknowledgments}

The study was supported by funding from the National Council of Science and Technology (CONACYT) Grant A1-S-8774 (CONACYT Ciencia Básica-Universidad de Guadalajara) assigned to JFMV. Also, CJH-B was a Ph.D. fellow at CONACYT-Mexico (\#301502). RB is supported by the US NIH.

\section{The authors declare no conflict of interest.}

\section{References}

1. Asano Y (2018): Systemic sclerosis. J Dermatol 45: 128-138.

2. Denton CP, Khanna D (2017): Systemic sclerosis. Lancet 390: 1685-1699.

3. Pattanaik D, Brown M, Postlethwaite BC, Postlethwaite AE (2015): Pathogenesis of Systemic Sclerosis. Front Immunol 6: 272 .

4. Pelaez-Ballestas I, Sanin LH, Moreno-Montoya J, et al. (2011): Epidemiology of the rheumatic diseases in Mexico.
A study of 5 regions based on the COPCORD methodology. J Rheumatol Suppl 86: 3-8.

5. Barnes J, Mayes MD (2012): Epidemiology of systemic sclerosis: incidence, prevalence, survival, risk factors, malignancy, and environmental triggers. Curr Opin Rheumatol 24: 165-170.

6. Selvi E, Tripodi SA, Catenaccio M, et al. (2003): Expression of macrophage migration inhibitory factor in diffuse systemic sclerosis. Ann Rheum Dis 62: 460-464.

7. Becker H, Willeke P, Schotte H, et al. (2008): Macrophage migration inhibitory factor may contribute to vasculopathy in systemic sclerosis. Clin Rheumatol 27: 1307-1311.

8. Ningyan G, Xu Y, Hongfei S, et al. (2015): The role of macrophage migration inhibitory factor in mast cell-stimulated fibroblast proliferation and collagen production. PLoS One 10: $\mathrm{e} 0122482$.

9. Kim JY, Kwok SK, Hur KH, et al. (2008): Up-regulated macrophage migration inhibitory factor protects apoptosis of dermal fibroblasts in patients with systemic sclerosis. Clin Exp Immunol 152: 328-335.

10. Schroder B (2016): The multifaceted roles of the invariant chain CD74 - more than just a chaperone. Biochim Biophys Acta 1863: 1269-1281.

11. Gore Y, Starlets D, Maharshak N, et al. (2008): Macrophage migration inhibitory factor induces $\mathrm{B}$ cell survival by activation of a CD74-CD44 receptor complex. J Biol Chem 283: 2784-2792.

12. Shi X, Leng L, Wang T, et al. (2006): CD44 is the signaling component of the macrophage migration inhibitory factor-CD74 receptor complex. Immunity 25: 595-606.

13. Schwartz V, Lue H, Kraemer S, et al. (2009): A functional heteromeric MIF receptor formed by CD74 and CXCR4. FEBS Lett 583: 2749-2757.

14. Corallo C, Paulesu L, Cutolo M, et al. (2015): Serum levels, tissue expression and cellular secretion of macrophage migration inhibitory factor in limited and diffuse systemic sclerosis. Clin Exp Rheumatol 33: S98-105.

15. Banos-Hernandez CJ, Navarro-Zarza JE, Bucala R, et al. (2019): Macrophage migration inhibitory factor polymorphisms are a potential susceptibility marker in systemic sclerosis from southern Mexican population: association with MIF mRNA expression and cytokine profile. Clin Rheumatol 38: 1643-1654.

16. van den Hoogen F, Khanna D, Fransen J, et al. (2013): 2013 classification criteria for systemic sclerosis: an American College of Rheumatology/European League against Rheumatism collaborative initiative. Arthritis Rheum 65: 2737-2747.

17. Chomczynski P, Sacchi N (1987): Single-step method of RNA isolation by acid guanidinium thiocyanate-phenol-chloroform extraction. Anal Biochem 162: 156-159.

18. Livak KJ, Schmittgen TD (2001): Analysis of relative gene expression data using real-time quantitative PCR and the 2(-Delta Delta C(T)) Method. Methods 25: 402-408.

19. Uhlen M, Fagerberg L, Hallstrom BM, et al. (2015): Proteomics. Tissue-based map of the human proteome. Science 347: 1260419.

20. Wu SP, Leng L, Feng Z, et al. (2006): Macrophage migration inhibitory factor promoter polymorphisms and the clinical expression of scleroderma. Arthritis Rheum 54: 3661-3669.

21. Jin J, Chou C, Lima M, et al. (2014): Systemic sclerosis is a complex disease associated mainly with immune regulatory and inflammatory genes. Open Rheumatol J 8: 29-42. 
22. Jankauskas SS, Wong DWL, Bucala R, et al. (2019): Evolving complexity of MIF signaling. Cell Signal 57: 76-88.

23. Karres I, Kremer JP, Dietl I, et al. (1998): Chloroquine inhibits proinflammatory cytokine release into human whole blood. Am J Physiol 274: R1058-1064.

24. Lindner R (2017): Invariant chain complexes and clusters as platforms for MIF signaling. Cells 6: 6.

25. Vincent FB, Lin E, Sahhar J, et al. (2018): Analysis of serum macrophage migration inhibitory factor and D-dopachrome tautomerase in systemic sclerosis. Clin Transl Immunology 7: e1042.

26. Hong Z, Jiang Z, Liangxi W, et al. (2004): Chloroquine protects mice from challenge with CpG ODN and LPS by decreasing proinflammatory cytokine release. Int Immunopharmacol 4: 223-234.

27. Petrovsky N, Socha L, Silva D, et al. (2003): Macrophage migration inhibitory factor exhibits a pronounced circadian rhythm relevant to its role as a glucocorticoid counter-regulator. Immunol Cell Biol 81: 137-143.

28. Calandra T, Roger T (2003): Macrophage migration inhibitory factor: a regulator of innate immunity. Nat Rev Immunol 3: 791-800.

29. Guo Y, Xiao P, Lei S, et al. (2008): How is mRNA expression predictive for protein expression? A correlation study on human circulating monocytes. Acta Biochim Biophys Sin (Shanghai) 40: 426-436.

30. Palanisamy V, Jakymiw A, Van Tubergen EA, et al. (2012): Control of cytokine mRNA expression by RNA-binding proteins and microRNAs. J Dent Res 91: 651-658.

31. Mino T, Takeuchi O (2013): Post-transcriptional regulation of cytokine mRNA controls the initiation and resolution of inflammation. Biotechnol Genet Eng Rev 29: 49-60.

32. Shimizu T (2005): Role of macrophage migration inhibitory factor (MIF) in the skin. J Dermatol Sci 37: 65-73.

33. Merk M, Baugh J, Zierow S, et al. (2009): The Golgi-associated protein $\mathrm{p} 115$ mediates the secretion of macrophage migration inhibitory factor. J Immunol 182: 6896-6906.

34. Zhao Y, Shimizu T, Nishihira J, et al. (2005): Tissue regeneration using macrophage migration inhibitory factor-impregnated gelatin microbeads in cutaneous wounds. Am J Pathol 167: 1519-1529.

35. Abe R, Shimizu T, Ohkawara A, Nishihira J (2000): Enhancement of macrophage migration inhibitory factor (MIF) expression in injured epidermis and cultured fibroblasts. Biochim Biophys Acta 1500: 1-9.

36. Pazyar N, Feily A, Yaghoobi R (2013): Macrophage migration inhibitory factor as an incriminating agent in dermatological disorders. Indian J Dermatol 58: 157.

37. McCoy SS, Reed TJ, Berthier CC, et al. (2017): Scleroderma keratinocytes promote fibroblast activation independent of transforming growth factor beta. Rheumatology (Oxford) 56: 1970-1981.

38. Yoshihisa Y, Rehman MU, Kondo T, Shimizu T (2016): Role of macrophage migration inhibitory factor in heat-induced apoptosis in keratinocytes. FASEB J 30: 3870-3877. 